\title{
Web application for galaxy-targeted follow-up of electromagnetic counterparts to gravitational wave sources ${ }^{\star}$
}

\author{
L. Salmon, L. Hanlon, R. M. Jeffrey, and A. Martin-Carrillo
}

School of Physics, University College Dublin, Belfield, Dublin 4, Ireland

e-mail: lana.salmon@ucdconnect.ie

Received 26 August 2019 / Accepted 11 December 2019

\begin{abstract}
The Laser Interferometer Gravitational Wave Observatory (LIGO) and Virgo Collaboration's Observing Run 3 has demanded the development of widely-applicable tools for gravitational wave follow-up. These tools must address the main challenges of the multimessenger era, namely covering large localisation regions and quickly identifying decaying transients. To address these challenges, we present a public web interface to assist astronomers in conducting galaxy-targeted follow-up of gravitational wave events by offering a fast and public list of targets post-gravitational wave trigger. After a gravitational wave trigger, the back-end galaxy retrieval algorithm identifies and scores galaxies based on the LIGO and Virgo computed probabilities and properties of the galaxies taken from the Galaxy List for the Advanced Detector Era V2 galaxy catalogue. Within minutes, the user can retrieve, download, and limit ranked galaxy lists from the web application. The algorithm and website have been tested on past gravitational wave events, and execution times have been analysed. The algorithm is being triggered automatically during Observing Run 3 and its features will be extended if needed. The web application was developed using the Python based Flask web framework.
\end{abstract}

Key words. gravitational waves - methods: observational - gamma-ray burst: general - telescopes

\section{Introduction}

The detection of GW170817 by the Advanced Laser Interferometer Gravitational Wave Observatory (LIGO; Aasi et al. 2015) and the Advanced Virgo detector (Acernese et al. 2014) triggered an international campaign of electromagnetic (EM) follow-up by ground and space-based observatories. This follow-up led to the detection of the almost coincident short-lived gammaray emission (Goldstein et al. 2017; Savchenko et al. 2017) and subsequent longer kilonova emission $\sim 2 \mathrm{kpc}$ from the galaxy NGC 4993 (Abbott et al. 2017a; Andreoni et al. 2017; Arcavi et al. 2017; Coulter et al. 2017; Cowperthwaite et al. 2017; Drout et al. 2017; Evans et al. 2017; Kilpatrick et al. 2017; McCully et al. 2017; Pian et al. 2017; Smartt et al. 2017; Tanvir et al. 2017; Valenti et al. 2017).

The gravitational wave $(\mathrm{GW})$ event is consistent with the signature expected from the inspiral and merger of two neutron stars (NS), accompanied by a gamma-ray burst (GRB). Neutron star mergers are thought to be the progenitors of short GRBs $(<2 s)$ as some short GRBs have been found in galaxies lacking in star formation (Berger 2014). The subsequent, more isotropic, transient (kilonova) is associated with radioactive decay of neutron rich material in the sub-relativistic outflow (Metzger 2017). Kilonova signatures have been found in the light curves of a handful of short GRBs (for a review, see Ascenzi et al. 2019), further strengthening their link to NS-NS mergers. This exciting event marked the end of Advanced LIGO's Observing Run 2 (O2; Abbott et al. 2019), which was followed by technical upgrades to both LIGO and Virgo in order to improve the sensitivity and range for Observing Run 3 (O3).

\footnotetext{
$\star$ The web application is freely available and publicly accessible at https://gwtool. watchertelescope.ie.
}

Even with the enhanced capabilities of the LIGO and Virgo Collaboration (LVC) during O3, which began in April 2019, the expected rate of NS-NS mergers that are detectable by the observatory (i.e. within about $170 \mathrm{Mpc}$ ) is only between one and 50 events per year (Abbott et al. 2018a). The rate of neutron starblack hole (NS-BH) merger events, which are also expected to produce EM counterparts, is highly uncertain but likely to be even lower (Abbott et al. 2018a).

With so few candidates expected, we need tools to identify electromagnetic counterparts as quickly as possible. This will allow us to probe the early-time physics of these events and answer open questions about the properties and progenitors of short GRBs (D'Avanzo et al. 2018; Lamb \& Kobayashi 2018; Ziaeepour 2018; Zhang et al. 2018), the neutron star equation of state (Abbott et al. 2018b; Annala et al. 2018; Bauswein et al. 2017; Coughlin et al. 2019a; Kiuchi et al. 2019; Radice et al. 2018; Raithel et al. 2018), $r$-process nucleosynthesis (Drout et al. 2017; Kasen et al. 2017; Pian et al. 2017), and cosmology (Abbott et al. 2017b; Hotokezaka et al. 2019; Vitale \& Chen 2018).

It is not simple to identify an EM counterpart to a merger event: the localisation region inferred from the GW signal is large (typically $\sim 20-1000 \mathrm{deg}^{2}$ Abbott et al. 2018a). Since the signal from the counterpart decays rapidly, the counterpart is often too faint to detect unless it can be localised quickly. Wide field of view telescopes ( $\mathrm{FoV}>1 \mathrm{deg}^{2}$ ) use tiling strategies to optimise coverage of the localisation regions (Andreoni et al. 2019; Coughlin et al. 2019b; Dobie et al. 2019; Goldstein et al. 2019); however, these regions are often too large for small field of view telescopes to survey quickly.

This paper presents a public web interface to a galaxy retrieval algorithm, which combines localisation information from LIGO/Virgo with galaxy positions from the Galaxy List for the Advanced Detector Era (GLADE; Dálya 2018) source 
Table 1. Features of existing and future wide and narrow field telescopes.

\begin{tabular}{llllll}
\hline \hline Telescope & \# Apertures & Aperture & $\begin{array}{l}\text { Field of view } \\
(\text { per telescope) } \\
\left(\mathrm{deg}^{2}\right)\end{array}$ & $\begin{array}{l}\text { \# Pointings for } \\
100 \mathrm{deg}^{2}\end{array}$ & Ref. \\
\hline Wide field telescopes & & & & \\
\hline ASSASN-1 & 4 & 0.14 & 4.5 & 23 & 1 \\
ATLAS & 2 & 0.5 & 29 & 4 & 2 \\
DECam & 1 & 4 & 3 & 34 & 3 \\
Evryscope & 27 & 0.061 & 8660 & $\mathrm{~N} / \mathrm{A}$ & 4 \\
GOTO & 4 & 0.4 & 5 & 20 & 5 \\
MASTER WFC & 16 & 0.082 & 24 & 5 & 6 \\
PANSTARRS-1 & 1 & 1.8 & 7 & 15 & 7 \\
BlackGEM ${ }^{(*)}$ & 4 & 0.6 & 2.7 & 38 & 9 \\
LSST $\left.{ }^{*}\right)$ & 1 & 8.4 & 9.6 & 3 & 10 \\
ZTF & 1 & 1.2 & 47 & & 11 \\
\hline Narrow field telescopes & 5 & & & 625 & 12 \\
\hline BOOTES & 1 & 0.6 & 0.16 & 625 & 13 \\
Liverpool Telescope & 1 & 2 & 0.076 & & \\
Watcher & 1 & 0.4 & 0.16 & & \\
\hline
\end{tabular}

Notes. The number of pointings is calculated based on the coverage of a square $10 \times 10 \mathrm{deg}^{2}$ region with tiles that don't overlap. ${ }^{(*)}$ Denotes future telescopes.

References. (1) Kochanek et al. (2017); (2) Tonry et al. (2018); (3) DePoy et al. (2008); (4) Law et al. (2015); (5) Dyer et al. (2018); (6) Lipunov et al. (2017); (7) Denneau et al. (2013); (8) Bloemen et al. (2015); (9) Ivezić et al. (2019); (10) Bellm et al. (2018), Graham et al. (2019), Masci et al. (2018); (11) Castro-Tirado et al. (2012); (12) Steele (2004); (13) French et al. (2004).

catalogue to provide the community with a ranked list of candidate galaxies for rapid follow-up observations after a GW detection. The paper is arranged as follows. Section 2 reviews current gravitational wave counterpart search strategies for optical and near-IR telescopes. Section 3 outlines the implementation and outputs of a galaxy ranking algorithm. The front-end web application which hosts the outputs of this algorithm is detailed in Sect. 4. Section 5 presents a discussion of the results of testing, use-cases, and future development.

\section{Review of search strategies}

\subsection{Strategies for wide field telescopes}

Many wide field survey telescopes and telescope networks have employed a tiling strategy, whereby their large fields of view and network capabilities allow for a large localisation region to be tiled in short times. The properties of a sample of these facilities compared to some narrow field telescopes can be seen in Table 1. For comparison, the resolution of typical LIGO/Virgo sky maps means that each pixel has an area of between 0.003 and $0.013 \mathrm{deg}^{2}$, much smaller than the field of view of both wide and narrow field facilities. Global networks such as the Global Rapid Advanced Network Devoted to the Multi-messenger Addicts (GRANDMA; Antier et al. 2019), the Global Relay of Observatories Watching Transients Happen (GROWTH; Andreoni et al. 2019; Coughlin et al. 2019b), and the Mobile Astronomical System of Telescope-Robots (MASTER; e.g. Lipunov et al. 2019) must coordinate the pointing strategies of their telescopes to maximise coverage and depth, and to make use of the extensive visibility of the telescopes in their network. Optical surveys such as the Zwicky Transient Facility (ZTF; Bellm et al. 2018; Graham et al. 2019; Masci et al. 2018) use a fixed grid on the sky to tile a region. This can assist candidate identification via image subtraction, but it does not optimise the coverage of a GW localisation region. The challenge faced by these observatories is to find the smallest number of tiles that can cover the entire localisation region, and/or swiftly identify the counterpart.

The tiling and scheduling strategies developed to face this challenge can be classified broadly into three approaches. The first is the probability-ranked observation of fixed-grid tiles (e.g. Coughlin \& Stubbs 2016; Ghosh et al. 2016). The second approach is the iterative placing of tiles to maximise the probability covered (e.g. Ghosh et al. 2016), and third is the detectability-based observation of tiles (e.g. Salafia et al. 2017). These algorithms are outlined, compared, and implemented in the gwemopt code (Coughlin et al. 2018, 2019c) along with various scheduling strategies. For a review of these strategies (see Coughlin et al. 2018; Rana et al. 2017).

\subsection{Strategies for narrow field telescopes}

Tiling strategies are impractical for narrow field of view telescopes to implement due to the large LVC localisation regions. The primary motivation behind this work is to provide the astrophysics community with a public web application to support galaxy-targeted searches of LVC localisation regions, more suited to telescopes with narrow fields of view. The main advantage of a galaxy-targeted strategy is the reduction in the number of pointings required by a factor of 10-100 (Gehrels et al. 2016). As an example, the $99 \%$ localisation regions of a sample of past GW events were covered with tiles of varying size, representing the fields of view of two current survey telescopes, ZTF and the Asteroid Terrestrial-impact Last Alert System (ATLAS), and a typical robotic telescope, such as the Watcher robotic telescope. Table 2 outlines the tiles required by each telescope to cover the entire region, as identified using the sky_tiling ${ }^{1}$ code. For comparison, Table 2 also shows the subset of tiles which contain galaxies identified using the algorithm outlined in Sect. 3 of this paper.

1 https://github.com/shaonghosh/sky_tiling.git 
Table 2. Comparison of the tiles required to cover a sample of LVC localisation regions with ZTF, ATLAS, and Watcher and the fraction of these tiles which contain galaxies.

\begin{tabular}{|c|c|c|c|c|c|c|}
\hline \multirow[b]{2}{*}{ GW event } & \multicolumn{2}{|c|}{ ZTF } & \multicolumn{2}{|r|}{ ATLAS } & \multicolumn{2}{|c|}{ Watcher } \\
\hline & Tiles & Galaxy tiles & Tiles & Galaxy tiles & Tiles & Galaxy tiles \\
\hline S190814bv & 58 & 58 & 98 & 98 & 12803 & 9248 \\
\hline S190828j & 44 & 42 & 72 & 69 & 7717 & 3677 \\
\hline S190910d & 191 & 190 & 334 & 333 & 210290 & 40273 \\
\hline S190923y & 120 & 118 & 205 & 203 & 27708 & 23834 \\
\hline S190924h & 30 & 30 & 51 & 51 & 6567 & 6222 \\
\hline S190930s & 100 & 100 & 175 & 174 & 24352 & 19512 \\
\hline S190930t & 779 & 764 & 1412 & 1391 & 210419 & 61563 \\
\hline
\end{tabular}

Notes. The $99 \%$ regions are covered in non-overlapping tiles calculated using the sky_tiling code. Tiles are equal in size to the telescope field of view, as stated in Table 1. The "Galaxy Tiles" column represents the number of tiles which contain galaxies identified by the algorithm described in this paper.

Rapid observation of fields of interest, preferably within one night, is required due to the fast evolving counterpart. For example, the kilonova associated with GW170817 decayed at a rate of $1.1 \mathrm{mag} / \mathrm{day}$ in the $r$ band (Valenti et al. 2017). The 22 galaxies identified to be within the $90 \%$ localisation region for this source could easily have been covered in one night of observing. The smaller number of fields also presents observers with the opportunity to allocate more time to observe each field and leads to quicker identification of counterparts.

An efficient way of determining the order in which to observe galaxies is to retrieve and rank the galaxies based on known properties of those galaxies and LVC provided probabilities. Various ranking algorithms have been outlined and implemented by telescopes and networks (e.g. Dobie et al. 2019; Ducoin et al. 2019; Kasliwal et al. 2017; Klingler et al. 2019; Rana \& Mooley 2019; Yang et al. 2019). Section 3 outlines a galaxy retrieval algorithm which utilises Arcavi et al. (2017).

\section{Galaxy retrieval algorithm}

The galaxy retrieval algorithm (Fig. 1) is implemented in Python 3.6.6 to interface with the Flask web framework and the ligo.skymap package. Figure 1 illustrates a flowchart of the algorithm which takes as input the sky map and GLADE galaxy catalogue and outputs a ranked galaxy list ordered by the probability of an association between the GW event and the galaxy.

\subsection{Inputs}

Each GW trigger contains the source classification, source localisation, and signal quality. The probability that the event originated from each source (binary black hole $(\mathrm{BBH})$, binary neutron star (BNS), NS-BH, MassGap, Terrestrial) is used to determine the most likely merger event that occurred. Also included in the trigger is the probability that the merger contains a NS, p(HasNS), and the probability that there is non-zero remnant matter, $p$ (HasRemnant). These parameters are used to determine the likelihood of an EM counterpart (Chatterjee et al. 2019). The signal quality is quantified by the false alarm rate (FAR), and the localisation and distance estimates of the source are included in a sky map file (Singer et al. 2016). The decision to followup an event is driven by the parameters included in the trigger, however the galaxy retrieval algorithm outlined in this section can respond to all events regardless of their classification.

\subsubsection{LVC sky map}

To choose which part of the sky is observed, and when, the "sky map" provided by the LVC is needed. Following a GW event, the BAYESTAR algorithm (Singer \& Price 2016) or the LALInference algorithm (LIGO Scientific Collaboration 2018; Veitch et al. 2015) outputs a sky map made up of pixels generated using the HEALPix projection (Górski et al. 2005). Each pixel in the HEALPix projection contains the following four values: the probability that the source is located in the pixel, a distance estimate, a standard deviation on the distance estimate, and a normalisation coefficient (Singer \& Price 2016). The header of the sky map also contains posterior mean of distance (DISTMEAN) and standard deviation of distance (DISTSTD) marginalised over the whole sky. For an overview of the parameters contained in the header and pixels, see Tables 1 and 2 of Singer et al. (2016). The LVC sky maps can be analysed in Python using the ligo. skymap package.

\subsubsection{GLADE galaxy catalogue}

In order to conduct galaxy-targeted follow-up, a galaxy catalogue is required. GLADE V2 $2^{2}$ (Dálya 2018) contains over 3.2 million objects, with over 2.9 million of these classified as a galaxy. It can be seen in Table 3 that over 1.6 million of these galaxies have corresponding distance and $B$ magnitude values. This subset is referred to as the filtered GLADE V2. A map of the filtered GLADE V2 galaxy density can be seen in Fig. 2.

We choose to use the filtered GLADE V2 catalogue in this work. When compiling GLADE V1, Dálya et al. (2016) used regression to estimate either or both of the redshift and $B$ magnitude for over 400000 galaxies. GLADE V2 omits these galaxies, and adds many new galaxies. While many of the newly added galaxies do not have associated $B$ magnitude or redshift values, we choose GLADE V2 as it includes the newest versions of its constituent catalogues, including the Two Micron All Sky Survey (2MASS; Skrutskie et al. 2006), HyperLEDA (Makarov et al. 2014), and the Gravitational Wave Galaxy Catalogue (GWGC; White et al. 2011).

The completeness of the filtered GLADE V2 catalogue is close to that of the filtered GLADE V1 catalogue which omits the galaxies whose parameters were estimated via regression. Completeness distances can be seen in Table 3. Completeness is measured by considering the cumulative blue luminosity of the galaxies within the filtered GLADE V1 \& V2 catalogues compared to the expected blue luminosity density from a homogeneous complete galaxy catalogue given by Kopparapu et al. (2008).

\subsection{Initial galaxy sample cut}

The algorithm developed for pre-processing of sky maps has been updated for the second half of $\mathrm{O} 3(\mathrm{O} 3 \mathrm{~b})$ to reduce execution time. From November 2019, V1 of this algorithm is used and prior to this V0 was used. Major updates include the analysis of only the $99 \%$ localisation region rather than the $50 \%, 90 \%$, and $99 \%$ regions, the increase of distance limits to \pm 5 DISTSTD from \pm DISTSTD and the removal of identification of the contour each galaxy lies within. The discussion of these changes,

\footnotetext{
2 http://glade.elte.hu/index.html
} 


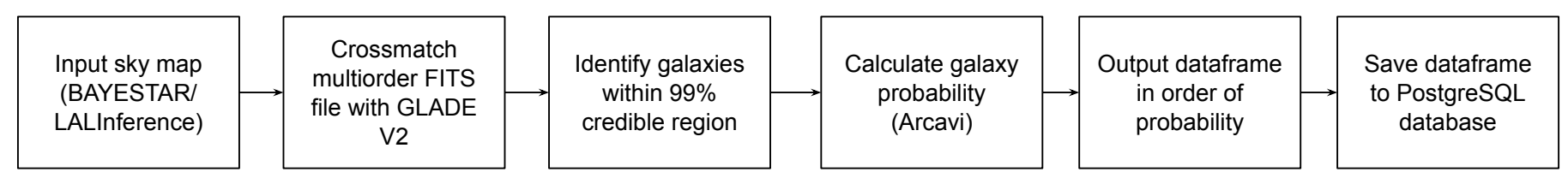

Fig. 1. Flowchart of the galaxy retrieval algorithm. The dataframes are saved as PostgreSQL database tables for each contour. This database is hosted on Amazon S3.

Table 3. Properties of GLADE V1 and GLADE V2.

\begin{tabular}{llllll}
\hline \hline Catalogue & \multirow{2}{*}{ \# Galaxies } & $\begin{array}{l}\text { \# Galaxies without } \\
\text { inferred distance }\end{array}$ & $\begin{array}{l}\text { \# Galaxies with known } \\
\text { distance and } B \text { magnitude }\end{array}$ & \multicolumn{2}{c}{ Completeness distance } \\
\cline { 3 - 6 } & & & & $100 \%$ & $50 \%$ \\
\hline GLADE V1 & 1918147 & 1490234 & 1490234 & $37 \mathrm{Mpc}$ & $164 \mathrm{Mpc}$ \\
\hline GLADE V2 & 2965718 & 2965718 & 1613030 & $37 \mathrm{Mpc}$ & $148 \mathrm{Mpc}$ \\
\hline
\end{tabular}

Notes. Completeness distance is measured by comparing the cumulative blue luminosity density within distinct distance limits to the luminosity density expected from a homogeneous complete galaxy catalogue, as outlined by Kopparapu et al. (2008).

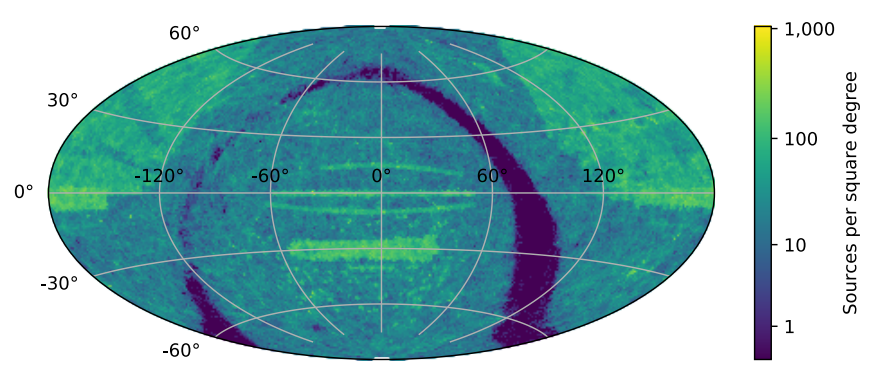

Fig. 2. Density of sources in the filtered GLADE V2 catalogue, displayed in RA and Dec. The colour scheme shows the number of galaxies per square degree; each pixel in the LVC data products has an area of between 0.003 and 0.013 square degrees. The varied coverage within the catalogue - especially along the Galactic plane - is obvious.

and details of V0 of the algorithm, can be seen in Sect. 5.3. V0 and V1 of the algorithm can be found on separate branches of GitHub $^{3}$.

In $\mathrm{V} 1$ of the algorithm, ligo.skymap is used for sky map I/O and also facilitates the crossmatching of a local version of the GLADE galaxy catalogue, stored as a hdf5 file, with the sky map. The galaxies within the $99 \%$ localisation regions and within DISTMEAN \pm 5 DISTSTD are identified using the ligo.skymap.crossmatch function. The coordinates, $B$ magnitudes, and distances to these galaxies, as taken from the filtered GLADE V2 catalogue, are stored in an array to be used in subsequent probability calculations. Figure 3 shows the galaxies from the filtered GLADE V2 catalogue identified to be within the $99 \%$ localisation regions for a variety of past GW events.

\subsection{Galaxy ranking}

The probability of association of the GW source with a given galaxy is calculated based on the approach outlined by Gehrels et al. (2016) and the prioritisation algorithm described by Arcavi et al. (2017), as follows:

1. The location probability measure is given as

$S_{\text {loc }}=p_{\text {loc }} p_{\text {dist }}$

\footnotetext{
3 https://github.com/Lanasalmon/HOGWARTs
}

The probability that the GW source is at a certain location, $p_{\text {loc }}$, is obtained from the pixel at the position of the galaxy in the sky map.

The distance to the merger computed by the LVC is contained in the pixel at the position of the galaxy. It is compared to the distance of the galaxy extracted from the filtered GLADE V2 galaxy catalogue to calculate the distance probability measure $p_{\text {dist }}$ :

$p_{\text {dist }}=N_{\text {dist }} \exp \left(\frac{-\left[D-\mu_{\text {dist }}\right]^{2}}{2 \sigma_{\text {dist }}^{2}}\right)$,

where $N_{\text {dist }}$ is a normalising factor, $\mu_{\text {dist }}$ is the distance estimate, and $\sigma_{\text {dist }}$ is the distance error computed by the BAYESTAR/LALInference algorithms and contained in the pixel at the galaxy's position sky map. $D$ is distance to the galaxy from the filtered GLADE V2 catalogue.

2. Short GRBs are found in the most massive galaxies, and $B$ luminosity is a proxy for galaxy mass (Berger 2014). Brighter galaxies are assigned a larger probability. The $B$-band luminosity is calculated using the apparent $B$ magnitude and distance from the filtered GLADE V2 catalogue. This is used to calculate the luminosity probability measure $S_{\text {lum }}$ :

$S_{\text {lum }}=\frac{L_{B}}{\sum L_{B}}$

3. The overall probability of the merger occurring in a galaxy is given by

$S=S_{\text {loc }} S_{\text {lum }}$.

This probability is calculated for all galaxies and then a score is computed by normalising the probabilities to add to 1 .

\subsection{Outputs}

The list of ranked galaxies is stored in a pandas dataframe which contains the name, Right Ascension, Declination, distance, $B$ magnitude, probability score, and cumulative score associated with each galaxy. The dataframes are saved as tables in a PostgreSQL database hosted on Amazon S3, where the database is accessible by the website via the Heroku command line tools. 


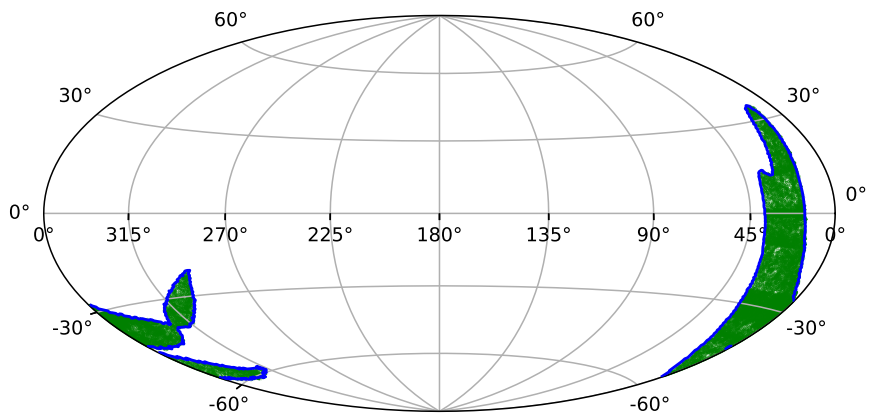

(a) S190814bv

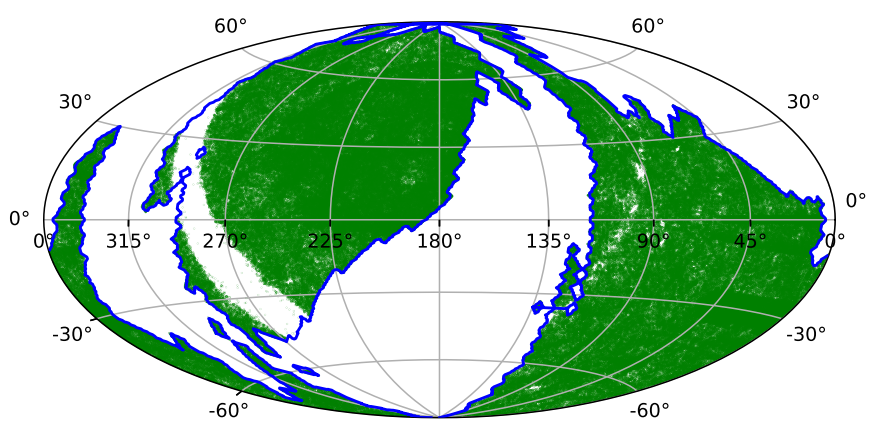

(c) S190901ap

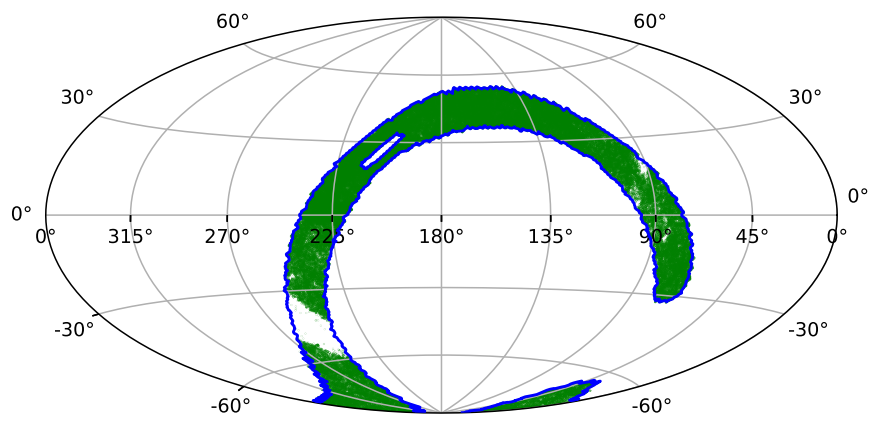

(e) S190923y

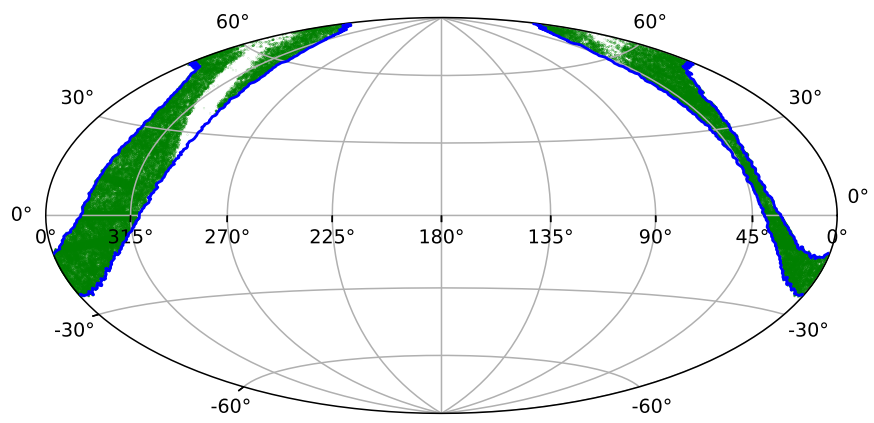

(g) S190930s

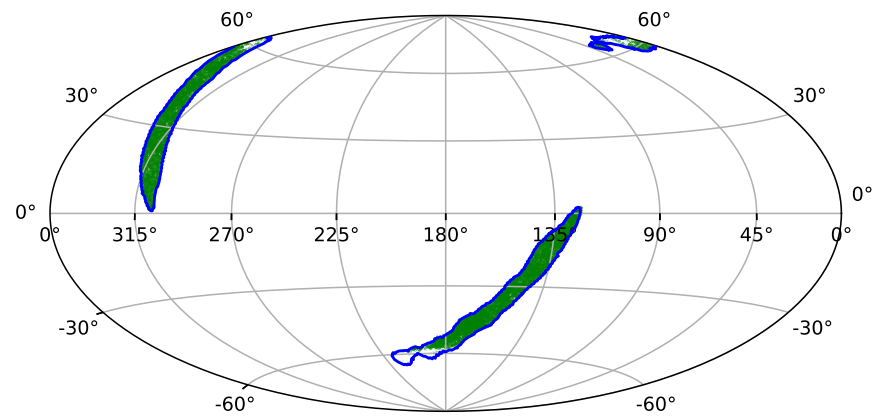

(b) $\mathrm{S} 190828 \mathrm{j}$

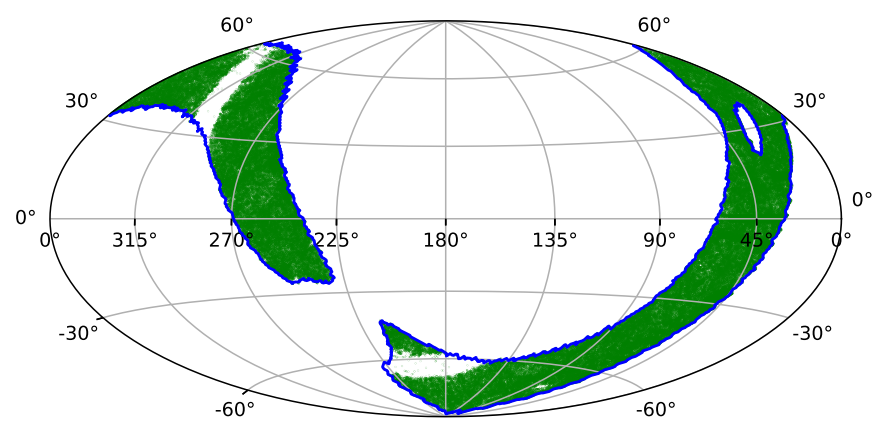

(d) S190910d

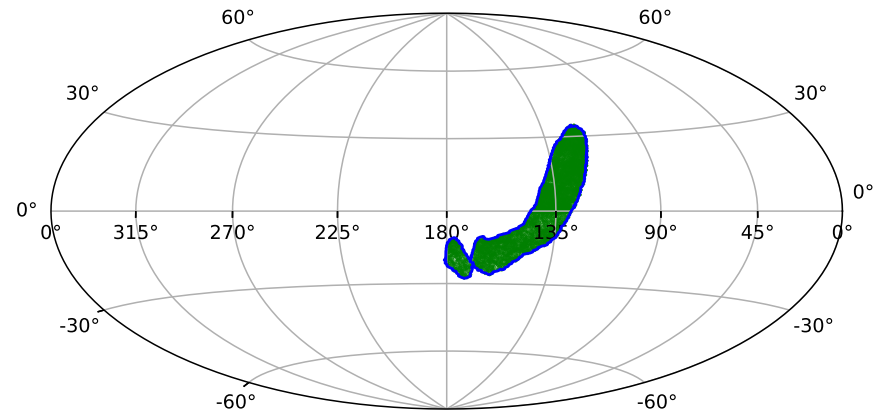

(f) $\mathrm{S} 190924 \mathrm{~h}$

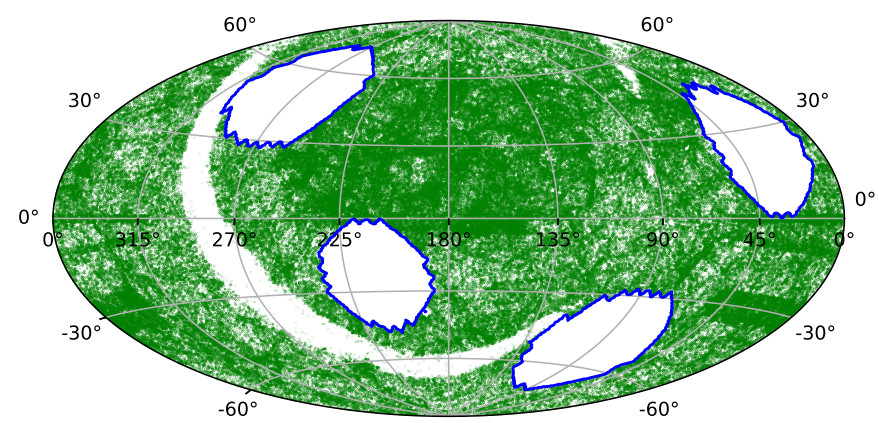

(h) $\mathrm{S} 190930 \mathrm{t}$

Fig. 3. $99 \%$ contour regions (blue lines) and galaxies from the filtered GLADE V2 catalogue (green points) which lie within these regions for a sample of initial sky maps for GW events from O3. We note that the galaxies are filtered based on the LVC distance estimate and error, within \pm 5 DISTSTD. These localisation regions are often irregularly shaped and have varied sizes. The uneven coverage of the filtered GLADE V2 catalogue is evident in these figures, especially in Fig. 3h. The galaxy density in each sky map is different due to the differing distance estimates (and completeness of the galaxy catalogue to that distance) and the magnitude of the corresponding standard deviations on distance.

\section{HOGWARTs web application}

The HOGWARTs front-end is a free and public web application which makes the outputs of the galaxy retrieval algorithm outlined in Sect. 3 publicly available. The web application also allows for further operations to be performed on the outputs for example, the galaxy list can be limited based on the visibility in a user's location, or on user-specified limiting magnitude.

To ensure fast and easy distribution of the outputs of the galaxy-ranking algorithm, a public web application was 


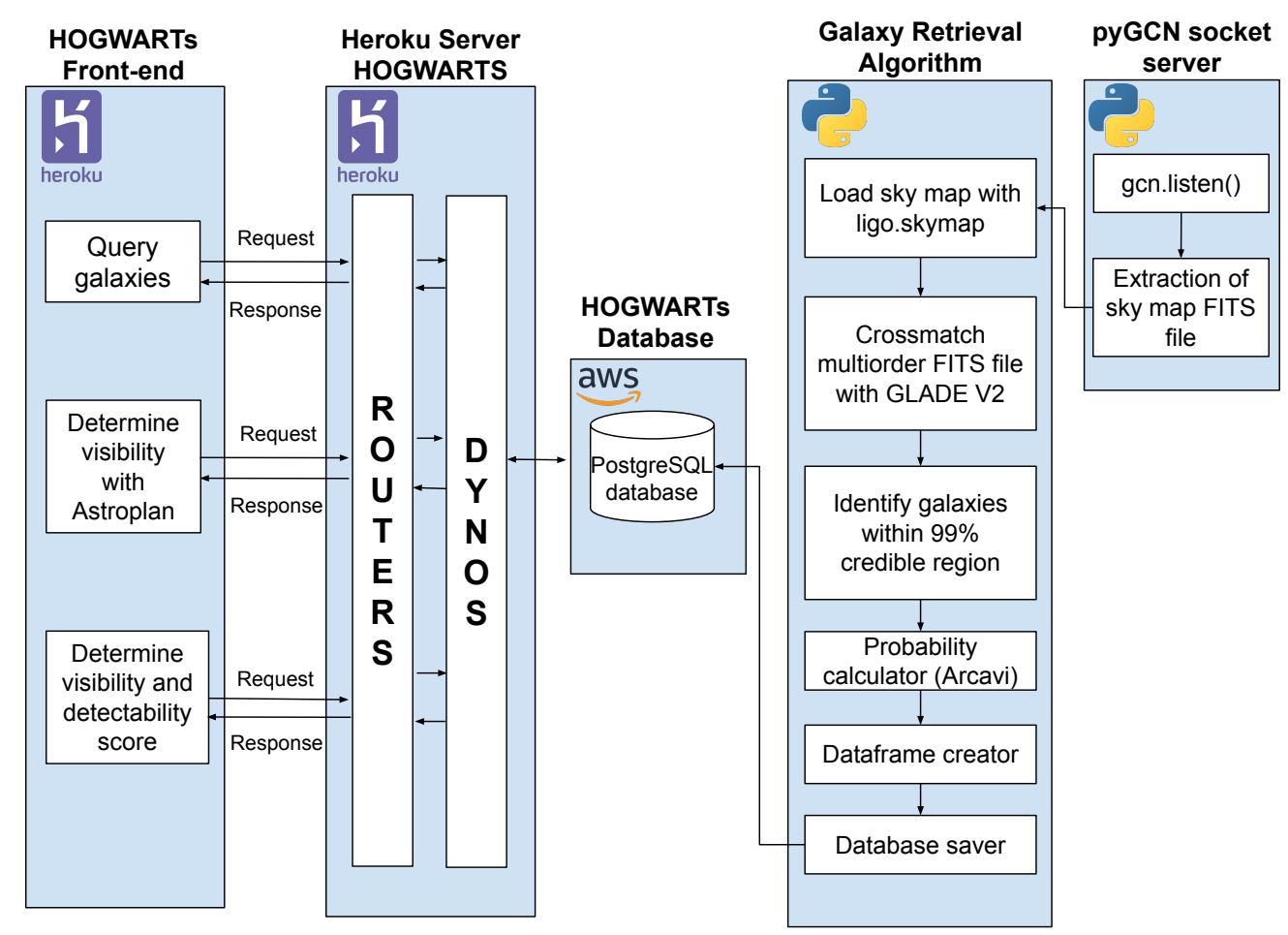

Fig. 4. Architecture of the HOGWARTs system. The galaxy retrieval algorithm saves the ranked galaxy lists for the $99 \%$ localisation region in a PostgreSQL database on Amazon S3. This database is connected to the HOGWARTs front-end on Heroku using the Heroku API. When a user requests the galaxy list, the website queries the database via Heroku routers and dynos to obtain the list of galaxies to render. Actions may be performed on this list in the Heroku back-end, including limiting the list based on visibility or detectability.

chosen. The only requirement for the user is a modern web browser with HTML-5 components and Javascript. This allows for universal instant access with little-to-no software installation. The distribution as a web application also allows for use by collaborations many of which have been established to tackle kilonova detection and identification during $\mathrm{O} 3$ using telescopes and networks. For example, to date the ElectromagNetic counterparts of GRAvitational wave sources at the VEry Large Telescope (ENGRAVE) collaboration, the GRAvitational Wave Inaf TeAm (GRAWITA; D'Avanzo et al. 2019a,b), the Gravitational Waves at the William Herschel Telescope collaboration (GW at WHT; Levan et al. 2019), and the GROWTH collaboration (Perley et al. 2019) have made use of the tool to conduct followup observations.

The HOGWARTs web application is implemented using the Flask web framework and is hosted on the cloud platform Heroku. The architecture of the HOGWARTs system can be seen in Fig. 4. Flask is a web framework which allows for web applications to be written in Python. Flask was chosen due to the flexibility of the framework. Heroku was chosen due to the ability to communicate via the command line with Amazon S3, GitHub, and the PostgreSQL database containing the results of the galaxy retrieval algorithm.

The back-end galaxy retrieval implementation uses pyGCN to act as a socket listening for GW alerts from the NASA Gamma-Ray Coordinates Network $(\mathrm{GCN})^{4}$ system. When an alert occurs, the algorithm immediately analyses the sky map and creates the database tables of galaxies corresponding to the 99\% localisation regions. These are saved on Amazon S3 and the algorithm updates the website to include a new menu option for this event.

\footnotetext{
4 https://gcn.gsfc.nasa.gov/lvc.html
}

\subsection{Inputs}

The HOGWARTs front-end web application renders the results of the galaxy retrieval algorithm in different ways depending on the user's requirements. The HOGWARTs web application currently supports three options with the possibility of adding more in the future. These are:

1. Retrieve the galaxy list (Fig. 5): The user chooses a GW source (e.g. GW170817) and a percentage localisation region $(99 \%, 90 \%$, or $50 \%)$.

2. Retrieve only the galaxies within a region that are visible from a specific location at a user-specified time: The user chooses a GW source (e.g. GW170817), a percentage localisation region $(99 \%, 90 \%$, or $50 \%)$, a longitude, latitude, limiting elevation, and time of observation. Alternatively, the user can choose their observatory from the predefined list (Boyden Observatory, La Palma, Paranal, and La Silla) if it is present. Astroplan (Astropy Collaboration 2018) is used to determine the galaxies visible to the user from astronomical twilight.

3. Retrieve only the galaxies that are visible from a specific location at a user-specified time including a detectability indicator: The user chooses from a menu as in option 2 , and also specifies the limiting magnitude. Alternatively, the user can choose their observatory from the predefined list as in option 2. An additional column indicates the detectability of a kilonova at that distance by comparing the minimum detectable source luminosity at that distance with that of a kilonova $\left(M_{\mathrm{KNmin}}=-17\right)$ at the same distance.

\subsection{Outputs}

The results webpage renders a table, ordered by probability score, containing a maximum of 100 galaxies to ensure 


\section{HOGWARTS Home About Contact}

\section{HOGWARTs}

\begin{tabular}{|c|c|}
\hline Home & $\begin{array}{l}\text { Hunt Of Gravitational Wave Areas for Rapid } \\
\text { Transients }\end{array}$ \\
\hline About & \multirow{3}{*}{$\begin{array}{l}\text { Retrieve galaxy list } \\
\text { Select a LIGONirgo source and a percentage confidence region to retrieve a list of galaxies } \\
\text { within that region: }\end{array}$} \\
\hline $\begin{array}{l}\text { Retrieve } \\
\text { galaxy list }\end{array}$ & \\
\hline $\begin{array}{l}\text { Retrieve } \\
\text { galaxy list } \\
\text { based on } \\
\text { location } \\
\end{array}$ & \\
\hline $\begin{array}{l}\text { Retrieve } \\
\text { galaxy list } \\
\text { based on } \\
\text { location } \\
\text { and } \\
\text { limiting } \\
\text { magnitude }\end{array}$ & $\begin{array}{l}\text { LIGONirgo source: } \\
\text { Percentage confidence region: } \\
99 \% \text {; }\end{array}$ \\
\hline & Retrieve galaxy list \\
\hline
\end{tabular}

Fig. 5. "Retrieve Galaxy List" input webpage at gwtool.watcher telescope.ie/retrieve_galaxies. The user chooses the GW source and percentage confidence region (99\%) to return the list of galaxies within those regions and a map of those galaxies.

rendering occurs in a timely manner. The table presents the galaxy name (from the GLADE V2 catalogue), probability score, Right Ascension, Declination, distance, $B$ magnitude, contour (if $\mathrm{V} 0$ of the algorithm was used), cumulative probability score, and an interactive Aladin DSS image of each galaxy, as shown in Fig. 6. A map of contour regions is also plotted. The HOGWARTs web application currently supports the download of the full and partial results tables as a json or ascii file. An extra column is visible for option 3 , indicating detectability of a possible kilonova associated with each galaxy. Additionally, the galaxies are plotted and a visibility plot is displayed for options 2 and 3.

\section{Discussion}

\subsection{User-testing and feedback}

Additional functionalities that were requested from collaborators were implemented. For example, the option to download the galaxy list as an ascii file was added. The web application has been tested on Windows and Mac OS using leading browsers.

\subsection{Evaluation of execution time}

The mean execution time of the back-end galaxy retrieval algorithm was evaluated using cProfile for past GW events. Typical execution times are between 20 and $30 \mathrm{~s}$, dependant upon the size (in MB) of the sky map FITS file, which in turn affects the time taken to download it. In addition to this execution time, $360 \mathrm{~s}$ is required to upload the new database and update the website on Heroku.

\subsection{V0 algorithm}

$\mathrm{V} 0$ of the pre-processing algorithm was used throughout the first half of $\mathrm{O} 3$ (O3a). This algorithm made use of healpy (Górski et al. 2005; Zonca et al. 2019) for sky map I/O and the skimage (van der Walt et al. 2014) find_contours method was used to identify the contours enclosing $99 \%, 90 \%$, and $50 \%$ of the probability within the integrated probability map. Each contour was treated separately, by querying GLADE in

\section{Contour 1: 10643 galaxies}

Contour 2: 5 galaxies

Number of galaxies: 10648 galaxies
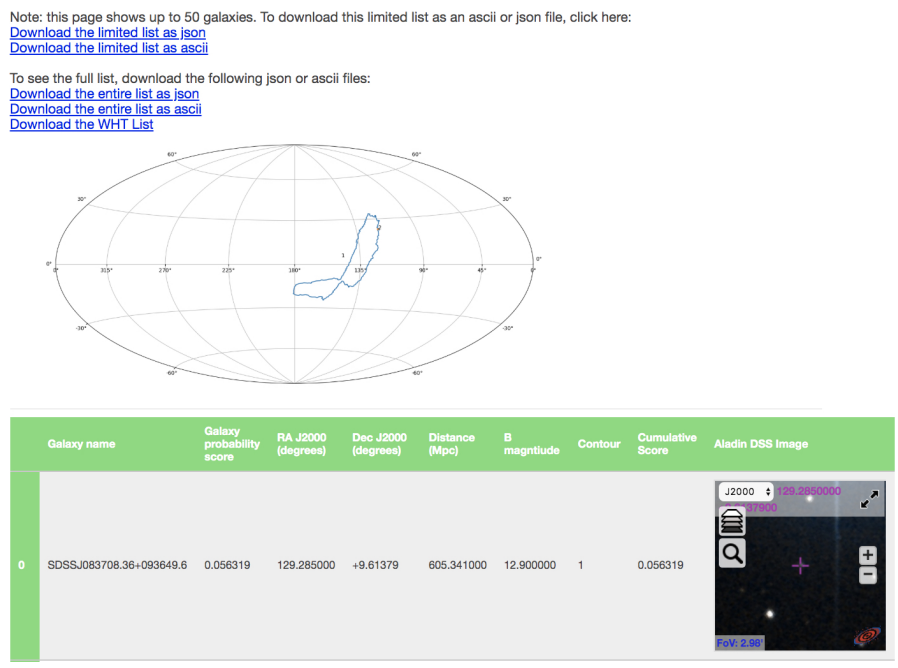

Fig. 6. Table rendered on the results webpage for the $99 \%$ confidence region for the updated sky map of $\$ 190924 \mathrm{~h}$. The tables on the results page are also accompanied by a map of contours, galaxies, and the option to download the tables as json or ascii files.

Vizier using Astropy (Astropy Collaboration 2018) with a circle enclosing each contour. The query was refined by only choosing galaxies within DISTMEAN \pm DISTSTD. Galaxies were determined to be within the contour using MOCPy, a Python package for analysing Multi-Order Coverage (MOC) maps. The coordinates, $B$ magnitudes, and distances to these galaxies, as taken from the filtered GLADE V2 catalogue, were stored in an array to be used in subsequent probability calculations and the contour within which each galaxy lay was noted.

Throughout O3a, it became clear that some of the features of this algorithm could be optimised or improved. Occasionally some galaxies which were ranked highly on the $99 \%$ list were not included in the $50 \%$ or $90 \%$ lists. This is due to high luminosity or distance probability measures, which therefore pushed galaxies outside the $50 \%$ or $90 \%$ regions up the $99 \%$ list. For a fair comparison of galaxies, we choose to only evaluate the $99 \%$ localisation region going forwards.

The range of galaxy distances considered is increased from DISTMEAN \pm DISTSTD to DISTMEAN \pm 5 DISTSTD to ensure that galaxies which may be ranked highly due to $B$ luminosity, but are outside of the $1 \sigma$ range, are included. This does not lengthen execution time and although it lengthens the galaxy lists, the majority of the extra galaxies included are ranked low due to their distances being significantly different from the mean distance in their pixels.

The identification of each galaxy's contour is computationally expensive as each contour needs to be considered separately. The identification of contours was initially implemented for easy identification of the galaxies which lie in contours that are visible to the user. However, it is noted that the visibility option on the website can be used instead of this feature, therefore removing the identification of contour regions is sensible for the reduction of execution time. Additionally, the release of multi-order sky maps within gravitational wave alerts and the addition of a crossmatching function within the ligo. skymap package allow for quick crossmatching of a local galaxy catalogue with a sky 
map. We are implementing this function from now on to reduce execution time.

\subsection{Comparison to existing follow-up tools}

The NED Gravitational Wave Follow-up (GWF) Service ${ }^{5}$ is a similar online tool which delivers a list of 2MASS galaxies within the $90 \%$ localisation region of a GW event minutes after trigger. It ranks based on the 2MASS Redshift Survey (2MRS) $K s$-band magnitude and does not make use of the information contained in the BAYESTAR/LALInference calculated probability and distance parameters. Throughout the start of O3, HOGWARTs galaxy lists have been broadly consistent with NED GWF galaxy lists. However, the NED GWF lists do not make a cut on distance, so provide all galaxies up to $200 \mathrm{Mpc}$. HOGWARTs lists also contain different candidate galaxies due to the use of a larger galaxy catalogue than NED.

The Global Relay of Observatories Watching Transients Happen (GROWTH) Target of Opportunity (ToO) Marshal has been developed by the GROWTH collaboration to coordinate follow-up observations of multi-messenger transients (Kasliwal et al. 2019). The Marshal responds to alerts by planning observations for a network of telescopes such as ZTF, Dark Energy Camera (DECam), and GROWTH-India and can send requests to robotic telescope queues. The tiling and scheduling features are beneficial for the network and for the wide field telescopes within that network, but the Marshal does not implement a galaxy identification or ranking feature.

\subsection{HOGWARTs in 03}

HOGWARTs has responded to gravitational wave alerts in $\mathrm{O} 3$ and the website has been updated accordingly. In particular, HOGWARTs was used to conduct follow-up observations of the NS-BH merger S190814bv. 74 galaxies which were identified within the $99 \%$ localisation region and ranked using the galaxy retrieval algorithm were followed up by the Telescopio Nazionale Galileo (TNG; D'Avanzo et al. 2019b,a), the WHT (Levan et al. 2019), the Nordic Optical Telescope (NOT; Heintz et al. 2019a,b), the Gamma-Ray Burst Optical/Near-Infrared Detector (GROND; Chen et al. 2019), and the Liverpool Telescope (LT; Perley et al. 2019). These follow-up observations took place within the ENGRAVE, GW at WHT, GROWTH, and GRAWITA collaborations. No candidate counterparts have yet been confirmed, however, the wide and deep coverage can place upper limits on electromagnetic counterparts from NS-BH mergers.

Each new NS-NS or NS-BH merger tests the galaxy-targeted strategy and the HOGWARTs system. It is therefore expected improvements will be made to the HOGWARTs system and perhaps additional functionality, such as scheduling and galaxy tiling tools, will be added to the current range of features available on the web application. However, it is currently considered that HOGWARTs is the first step in an individual's pipeline, allowing the individual to choose the scheduling that best suits their observatory.

Throughout $\mathrm{O} 3 \mathrm{~b}$ it is expected that further improvements will be made to the system, including the possible transfer of the web application from Heroku to a private server to reduce the time it takes to update the website post-trigger. Further

\footnotetext{
5 The NASA/IPAC Extragalactic Database (NED) is operated by the Jet Propulsion Laboratory, California Institute of Technology, under contract with the National Aeronautics and Space Administration.
}

modifications to the distance limits are expected to consider the $3 \mathrm{D}$ credible region rather than the $2 \mathrm{D}$ credible region within distance limits.

\section{Conclusions}

HOGWARTs is a web application for retrieving lists of candidate galaxies to observe in GW localisation regions. This publicly accessible tool contributes to the critical need for tools to assist astronomers in conducting GW follow-up. This website is easily accessible worldwide and the code is open-source. Collaborations and single-users alike can make use of this tool to schedule their observations swiftly post-trigger and incorporate the website into their existing pipelines, tools, and processes. The back-end algorithm and website have been tested on past LVC sky maps and execution times have been analysed. With perhaps tens of NS-NS mergers expected during O3, there will be opportunities to test and improve this strategy and extend the functionality of the website over the coming months.

Acknowledgements. LS acknowledges the Irish Research Council Postgraduate Scholarship No GOIPG/2017/1525. We are grateful to Alberto Castro-Tirado, Morgan Fraser, James Gillanders, Kate Maguire, Owen McBrien, Stephen Smartt, and Brian Van Soelen for their useful feedback on the HOGWARTs web application. We would like to thank the anonymous referee for their constructive comments that helped us improve the algorithm and content of the manuscript.

\section{References}

Aasi, J., Abbott, B., Abbott, R., et al. 2015, Class. Quant. Grav., 32, 074001 Abbott, B. P., Abbott, R., Abbott, T., et al. 2017a, ApJ, 848, L12 Abbott, B. P., Abbott, R., Abbott, T. D., et al. 2017b, Nature, 551, 85 Abbott, B. P., Abbott, R., Abbott, T., et al. 2018a, Liv. Rev. Relativ., 21, 3 Abbott, B., Abbott, R., Abbott, T., et al. 2018b, Phys. Rev. Lett., 121, 161101 Abbott, B. P., Abbott, R., Abbott, T., et al. 2019, ApJ, 875, 161

Acernese, F., Agathos, M., Agatsuma, K., et al. 2014, Class. Quant. Grav., 32, 024001

Andreoni, I., Ackley, K., Cooke, J., et al. 2017, PASA, 34, e069

Andreoni, I., Goldstein, D. A., Anand, S., et al. 2019, ApJ, 881, L16

Annala, E., Gorda, T., Kurkela, A., \& Vuorinen, A. 2018, Phys. Rev. Lett., 120, 172703

Antier, S., Agayeva, S., Aivazyan, V., et al. 2019, MNRAS, stz3142

Arcavi, I., Hosseinzadeh, G., Howell, D. A., et al. 2017, Nature, 551, 64

Ascenzi, S., Coughlin, M. W., Dietrich, T., et al. 2019, MNRAS, 486, 672

Astropy Collaboration (Price-Whelan, A., et al.) 2018, AJ, 156, 123

Bauswein, A., Just, O., Janka, H.-T., \& Stergioulas, N. 2017, ApJ, 850, L34

Bellm, E. C., Kulkarni, S. R., Graham, M. J., et al. 2018, PASP, 131, 018002

Berger, E. 2014, ARA\&A, 52, 43

Bloemen, S., Groot, P., Nelemans, G., \& Klein-Wolt, M. 2015, in The BlackGEM Array: Searching for Gravitational Wave Source Counterparts to Study UltraCompact Binaries, ASP Conf. Ser., 496, 254

Castro-Tirado, A. J., Jelínek, M., Gorosabel, J., et al. 2012, Astron. Soc. India Conf. Ser., 7, 313

Chatterjee, D., Ghosh, S., Brady, P. R., et al. 2019, ApJ, submitted [arXiv:1911.00116]

Chen, T.-W., Nicuesa Guelbenzu, A., Fraser, M., et al. 2019, GCN Circ., 25372 Coughlin, M., \& Stubbs, C. 2016, Exp. Astron., 42, 165

Coughlin, M. W., Tao, D., Chan, M. L., et al. 2018, MNRAS, 478, 692

Coughlin, M. W., Dietrich, T., Margalit, B., \& Metzger, B. D. 2019a, MNRAS, 489, L91

Coughlin, M. W., Ahumada, T., Anand, S., et al. 2019b, ApJ, 885, L19

Coughlin, M. W., Antier, S., Corre, D., et al. 2019c, MNRAS, 489, 5775

Coulter, D., Foley, R., Kilpatrick, C., et al. 2017, Science, 358, 1556

Cowperthwaite, P., Berger, E., Villar, V., et al. 2017, ApJ, 848, L17

Dálya, G. 2018, MNRAS, 479, 2374

Dálya, G., Frei, Z., Galgoczi, G., Raffai, P., \& de Souza, R. S. 2016, VizieR Online Data Catalog: VII/275

D’Avanzo, P., Campana, S., Salafia, O., et al. 2018, A\&A, 613, L1

D'Avanzo, P., Melandri, A., Izzo, L., et al. 2019a, GCN Circ., 25331

D'Avanzo, P., Rossi, A., Greco, G., et al. 2019b, GCN Circ., 25361

Denneau, L., Jedicke, R., Grav, T., et al. 2013, PASP, 125, 357 
DePoy, D. L., Abbott, T., Annis, J., et al. 2008, in The Dark Energy Camera (DECam), SPIE Conf. Ser., 7014, 70140E

Dobie, D., Murphy, T., Kaplan, D., et al. 2019, PASA, 36, e019

Drout, M., Piro, A., Shappee, B., et al. 2017, Science, 358, 1570

Ducoin, J. G., Corre, D., Leroy, N., \& Floch, E. L. 2019, ArXiv e-prints [arXiv:1911.05432]

Dyer, M. J., Dhillon, V. S., Littlefair, S., et al. 2018, SPIE Conf. Ser., 10704 107040C

Evans, P., Cenko, S., Kennea, J. A., et al. 2017, Science, 358, 1565

French, J., Hanlon, L., McBreen, B., et al. 2004, in Gamma-Ray Bursts: 30 Years of Discovery, eds. E. Fenimore, \& M. Galassi, Am. Inst. Phys. Conf. Ser., 727 741

Gehrels, N., Cannizzo, J. K., Kanner, J., et al. 2016, ApJ, 820, 136

Ghosh, S., Bloemen, S., Nelemans, G., Groot, P. J., \& Price, L. R. 2016, A\&A 592, A82

Goldstein, A., Veres, P., Burns, E., et al. 2017, ApJ, 848, L14

Goldstein, D. A., Andreoni, I., Nugent, P. E., et al. 2019, ApJ, 881, L7

Górski, K. M., Hivon, E., Banday, A. J., et al. 2005, ApJ, 622, 759

Graham, M. J., Kulkarni, S., Bellm, E. C., et al. 2019, PASP, 131, 078001

Heintz, K., Malesani, D., Leloudas, G., et al. 2019a, GCN Circ., 25376

Heintz, K. E., Malesani, D., Selsing, J., et al. 2019b, GCN Circ., 25325

Hotokezaka, K., Nakar, E., Gottlieb, O., et al. 2019, Nat. Astron., 3, 940

Ivezić, Ž., Kahn, S. M., Tyson, J. A., et al. 2019, ApJ, 873, 111

Kasen, D., Metzger, B., Barnes, J., Quataert, E., \& Ramirez-Ruiz, E. 2017, Nature, 551, 80

Kasliwal, M., Nakar, E., Singer, L., et al. 2017, Science, 358, 1559

Kasliwal, M., Cannella, C., Bagdasaryan, A., et al. 2019, PASP, 131, 038003

Kilpatrick, C. D., Foley, R. J., Kasen, D., et al. 2017, Science, 358, 1583

Kiuchi, K., Kyutoku, K., Shibata, M., \& Taniguchi, K. 2019, ApJ, 876, L31

Klingler, N., Kennea, J., Evans, P., et al. 2019, ApJS, 245, 15

Kochanek, C., Shappee, B., Stanek, K., et al. 2017, PASP, 129, 104502

Kopparapu, R. K., Hanna, C., Kalogera, V., et al. 2008, ApJ, 675, 1459

Lamb, G. P., \& Kobayashi, S. 2018, MNRAS, 478, 733

Law, N. M., Fors, O., Ratzloff, J., et al. 2015, PASP, 127, 234

Levan, A., Fraser, M., Jonker, P., et al. 2019, GCN Circ., 25332
LIGO Scientific Collaboration 2018, LIGO Algorithm Library - LALSuite, free software (GPL)

Lipunov, V., Gorbovskoy, E., Kornilov, V., et al. 2017, ApJ, 850, L1

Lipunov, V., Gorbovskoy, E., Kornilov, V., et al. 2019, GCN Circ., 24236

Makarov, D., Prugniel, P., Terekhova, N., Courtois, H., \& Vauglin, I. 2014, A\&A, 570, A13

Masci, F. J., Laher, R. R., Rusholme, B., et al. 2018, PASP, 131, 018003

McCully, C., Hiramatsu, D., Howell, D. A., et al. 2017, ApJ, 848, L32

Metzger, B. D. 2017, Liv. Rev. Relativ., 20, 3

Perley, D. A., Copperwheat, C. M., Malesani, D. B., \& Levan, A. 2019, GCN Circ., 25328

Pian, E., d'Avanzo, P., Benetti, S., et al. 2017, Nature, 551, 67

Radice, D., Perego, A., Zappa, F., \& Bernuzzi, S. 2018, ApJ, 852, L29

Raithel, C. A., Özel, F., \& Psaltis, D. 2018, ApJ, 857, L23

Rana, J., \& Mooley, K. P. 2019, ApJ, submitted [arXiv:1904.07335]

Rana, J., Singhal, A., Gadre, B., Bhalerao, V., \& Bose, S. 2017, ApJ, 838, 108

Salafia, O. S., Colpi, M., Branchesi, M., et al. 2017, ApJ, 846, 62

Savchenko, V., Ferrigno, C., Kuulkers, E., et al. 2017, ApJ, 848, L15

Singer, L. P., \& Price, L. R. 2016, Phys. Rev. D, 93, 024013

Singer, L. P., Chen, H.-Y., Holz, D. E., et al. 2016, ApJS, 226, 10

Skrutskie, M., Cutri, R., Stiening, R., et al. 2006, AJ, 131, 1163

Smartt, S., Chen, T.-W., Jerkstrand, A., et al. 2017, Nature, 551, 75

Steele, I. 2004, Astron. Nachr., 325, 519

Tanvir, N., Levan, A., González-Fernández, C., et al. 2017, ApJ, 848, L27

Tonry, J., Denneau, L., Heinze, A., et al. 2018, PASP, 130, 064505

Valenti, S., David, J., Yang, S., et al. 2017, ApJ, 848, L24

van der Walt, S., Schönberger, J. L., Nunez-Iglesias, J., et al. 2014, ArXiv e-prints [arXiv:1407.6245]

Veitch, J., Raymond, V., Farr, B., et al. 2015, Phys. Rev. D, 91, 042003

Vitale, S., \& Chen, H.-Y. 2018, Phys. Rev. Lett., 121, 021303

White, D. J., Daw, E., \& Dhillon, V. 2011, Class. Quant. Grav., 28, 085016

Yang, S., Sand, D. J., Valenti, S., et al. 2019, ApJ, 875, 59

Zhang, B.-B., Zhang, B., Sun, H., et al. 2018, Nat. Commun., 9, 447

Ziaeepour, H. 2018, MNRAS, 478, 3233

Zonca, A., Singer, L., Lenz, D., et al. 2019, J. Open Source Softw., 4, 1298 